\title{
Applying Model Based Systems Engineering to NASA's Space Communications Networks
}

\author{
Kul Bhasin, Patrick Barnes, Jessica Reinert, Bert Golden, \\ National Aeronautics Space Administration \\ Glenn Research Center \\ Cleveland, Ohio
}

\begin{abstract}
System engineering practices for complex systems and networks now require that requirement, architecture, and concept of operations product development teams, simultaneously harmonize their activities to provide timely, useful and cost-effective products. When dealing with complex systems of systems, traditional systems engineering methodology quickly falls short of achieving project objectives. This approach is encumbered by the use of a number of disparate hardware and software tools, spreadsheets and documents to grasp the concept of the network design and operation. In case of NASA's space communication networks, since the networks are geographically distributed, and so are its subject matter experts, the team is challenged to create a common language and tools to produce its products. Using Model Based Systems Engineering methods and tools allows for a unified representation of the system in a model that enables a highly related level of detail. To date, Program System Engineering (PSE) team has been able to model each network from their top-level operational activities and system functions down to the atomic level through relational modeling decomposition. These models allow for a better understanding of the relationships between NASA's stakeholders, internal organizations, and impacts to all related entities due to integration and sustainment of existing systems.

Understanding the existing systems is essential to accurate and detailed study of integration options being considered. In this paper, we identify the challenges the PSE team faced in its quest to unify complex legacy space communications networks and their operational processes. We describe the initial approaches undertaken and the evolution toward model based system engineering applied to produce Space Communication and Navigation (SCaN) PSE products. We will demonstrate the practice of Model Based System Engineering applied to integrating space communication networks and
\end{abstract}

the summary of its results and impact. We will highlight the insights gained by applying the Model Based System Engineering and provide recommendations for its applications and improvements.

\section{Keywords-NASA, Model Based Systems \\ Engineering, Space Communications, Ground Network Integration}

\section{INTRODUCTION}

In 2006, NASA administration mandated the centralization of the management of NASA's Space Communications and Navigation (SCaN) networks: the Near Earth Network (NEN), the Space Network (SN), and the Deep Space Network (DSN). Currently, these networks provide communication and tracking services to user missions through NASA SCaN Program [1]. The networks have evolved over a number of years and have utilized the technologies available during the implementation and upgrade periods. The recent developments in hardware and software technologies have the potential to integrate the current configuration of loosely coupled networks into a single, unified, integrated network while providing savings in lifecycle costs. An Integrated Network Architecture (INA) trade study team was established and comprised members with diverse skill sets from various networks system engineering organizations. The INA team studied various options on the integration of the networks and has summarized its finding elsewhere [2]. Initially they used document based system engineering. It was used to document information and as well as create models of architecture concepts. Data was stored in a common repository accessible by INA team members. The process was characterized by the generation of text-based specifications and design documents, in hardcopy or electronic file format, that are then exchanged between customers, users, developers and testers [1]. Emphasis was placed on controlling the documentation and ensuring it is valid, complete, and consistent and that the developed system complies with the documentation. 
Because the information was spread across multiple documents, the completeness, consistency and relationships among requirements, design, engineering analysis, and test information could be difficult to assess. The INA team found it difficult to understand particular aspects of the networks to perform traceability and change impact assessments. It became difficult to maintain or reuse the system requirements and design information for an evolving integrated network high-level architecture design and operations. They then embarked on applying the Model Based System Engineering (MBSE) to its trade options analysis for the development of INA.

The following sections describe the approach taken to set up the MBSE methodology. Then the details of the use of document based systems engineering, transition and application of MBSE to the trade study analysis of the INA. We discuss the current status and present the conclusion.

\section{ESTABLISHING THE MOdel BASED \\ ENGINEERING METHODOLOGY}

The use of MBSE started with the rendering of the legacy network architectures and operations since the network were designed and implemented in the era of meager documentation, much of which was no longer available. However, the tool being used to develop the architecture was not found suitable for the INA trade study work because the tool required updates to individual diagrams rather than allowing an element to be updated and having all the diagrams updated simultaneously which resulted in significant rework for even small architecture changes. A small trade study was conducted [3] to determine the MBSE tool that would be used. Several tools were evaluated for applying MBSE to study and develop the INA. These tools comprised of MagicDraw, Rhapsody, Enterprise Architect, CORE, DOORS, CRADLE and Artisan Studio. The tool identified was No Magic's MagicDraw. The INA team was trained and the modelers were charged to decide what framework/language to use. The modelers started off using Department of Defense Architecture Framework (DoDAF) views [4] but eventually migrated to SysML [5] in favor of a less constrained modeling environment.

MBSE requires upfront investment in tools and training. Document based practices were supported while establishing the new MBSE processes to perform system engineering functions. Several modeling methodologies and languages such UML2 - Unified Modeling Language 2 (Software Engineers), SysML - System Modeling Language (Systems Engineers), AADL - Architecture Analysis and Design Language (Society of Automotive Engineers), BPMN/UML - Business Process
Modeling Notation/Unified Modeling Language (Business Analysts) were reviewed.

\section{IMPLEMENTING MBSE FOR SPACE}

COMMUNICATION NETWORK INTEGRATION

The INA team started out documenting the three NASA space communication networks based on the DoDAF using Microsoft office tools. The same tools were used to produce the INA options and that is when they begin to notice the need for the MBSE. Although, this approach provided an introduction to existing Networks for those working on integration activities, some of the INA team members started working on the implementation of MBSE to better quantify potential benefits of its use. Below are the details of these steps which also include how MBSE was finally applied to the INA trade studies.

\section{A. Documenting the Integrated Network \\ Architecture}

The INA team's first step was to examine the current opertional processes and software systems of the Operational Networks. The INA team, with the ultimate goal of designing an integrated ("To-Be") architecture for the network, needed to understand the details of each operational network. To do so, network characteristics were tracked in vast spreadsheets, terminology and definitions were put into excel format and discussed at length, "as-is" PowerPoint diagrams were created; all to better understand the characteristics of the networks.

While Microsoft PowerPoint was used to create architecture diagrams for presentation (see Fig. 1), it lacks the ability to create levels of archtiecture information, reuse of elements throughout seveal diagrams, and maintains a single data element repository, to mention a few short comings. The diagram below (see Fig. 2), shows a relatively similar diagram to Fig. 1, but now represented using SysML in MagicDraw. This diagram (Fig. 2) can convey all of the information shown in Fig. 1 and more, while also utilizing the MBSE model architecture to link this diagram with all other diagrams in the model.

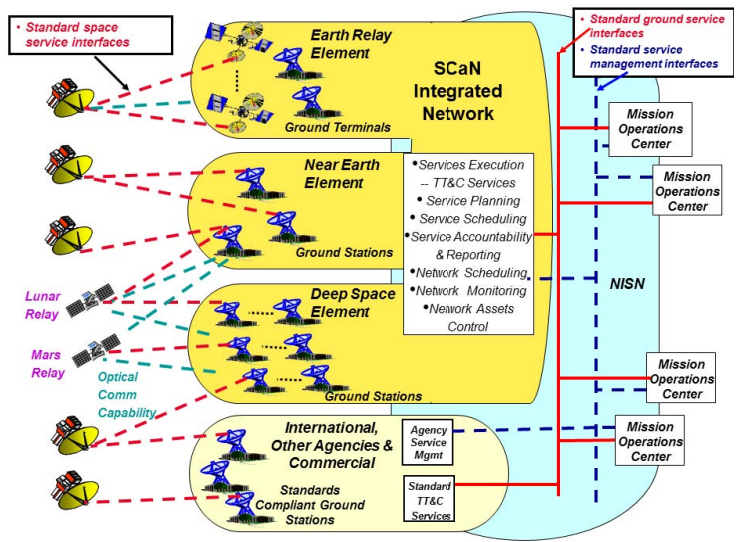

Fig. 1: SCaN Network To-Be Architecture 


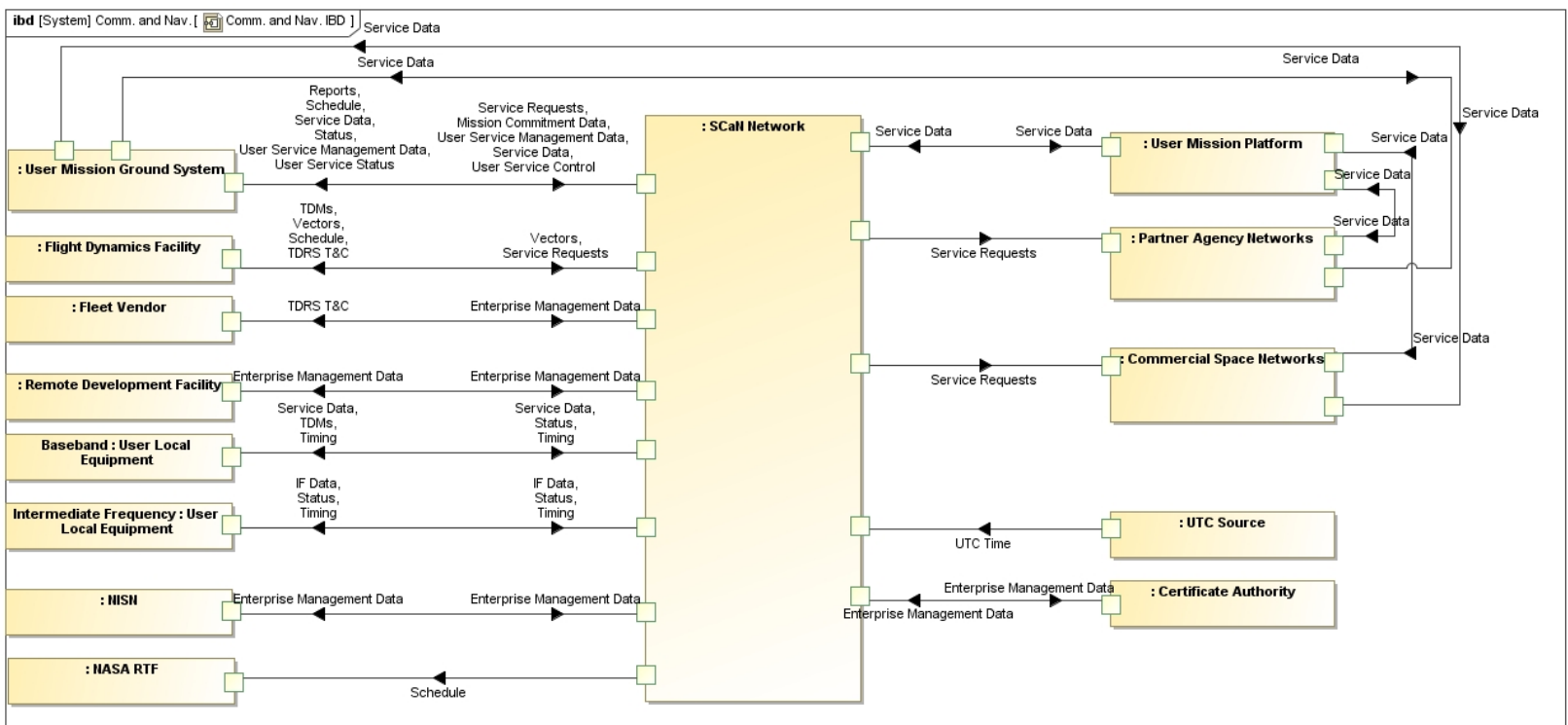

Fig. 2: SCaN Communications and Navigation Internal Block Diagram (IBD)

The issue, as mentioned above, with using PowerPoint as the medium for communicating system architecture is that each diagram ends up being a separate unit; no relation to other diagrams. As shown in Fig. 3, a block exists on the diagram named "INOC". In Fig. 4, an "INOC" block also exists, though the two are unrelated [1]. These diagrams show two different views of the same block. The block has some of the same parts, but the hardware architecture diagram shows more detailed information about the IT infrastructure in that block which the system architecture diagram does not show.

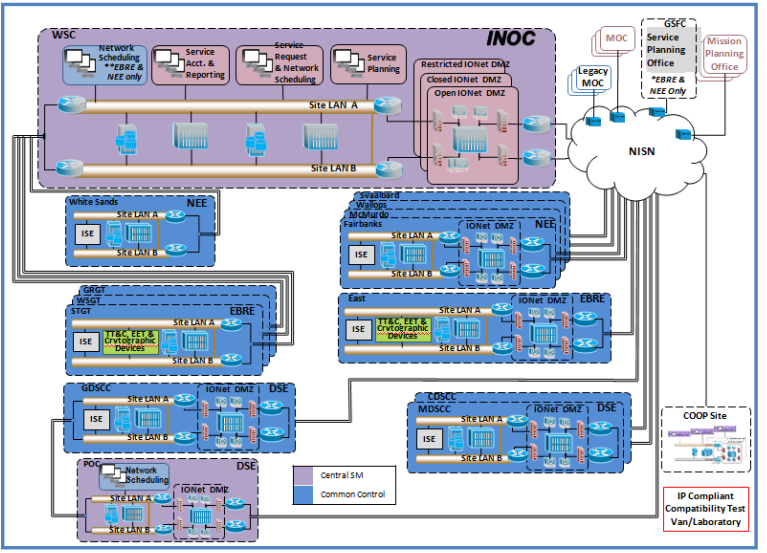

Fig. 3: Integrated Network Management (INM) System Architecture

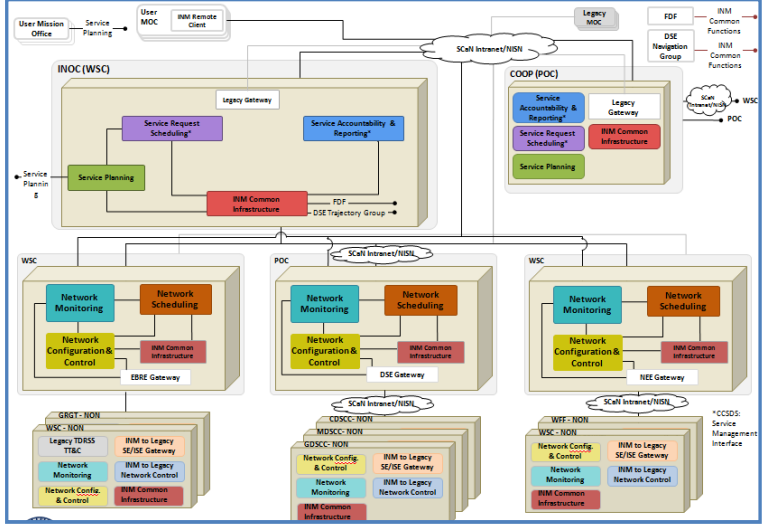

Fig. 4: INM Hardware Architecture

\section{B. Transitioning to MBSE}

Weekly tag-ups were held between the modelers while the developed a modeling standard which would be used for all proceeding architecture modeling. Numerous revisions were made to the application of the SysML methodology by the Integrated Network Architecture modelers while deciding what would best fit the needs of the INA team.

The INA modeling team split into two subgroups which divided modeling tasks for the trade study. Some of the INA team modeled the software data systems of the INA network, while the rest modeled Operational Process Flows (OPF) for the operations of the network. In the diagram below, (Fig. 5), a portion of the Network Control Operational Process Flow for Option One (the INA trade study detailed several options to investigate during the study) nominal mission event. Some of the methodologies sown in this diagram are no longer 
used by the INA team as a result of our weekly meetings.

Fig. 5 represents what was the INA modelers' first attempt at MBSE modeling using SysML. Using MBSE allowed the INA team to consolidate what would have been dozens of documents and spreadsheets into one cohesive model which could be represented visually while linking all elements on each diagram. If a modeler needed to change the name of a block for a future system, that change could be applied in once place and the MBSE tool would resonate that change throughout the model. The change also provided the unexpected benefit of accelerating the INA team members' understanding of the trade studies. Instead of spending a week or more reading systems engineering documents, s/he could review the model, a single point of information, to quickly come up to speed on all current architecture design used by the INA team.
INA model. The evolution of MBSE for the INA team has led to increasingly complex diagrams that contain a greater level of detail. The diagram below is a "rolled up" version of a dozen sub interface diagrams that exist in the overall architecture model.

The MBSE work was also adopted by the baseline book teams. The Architecture Definition Document (ADD) team has been quick to adopt the MBSE methodologies used by the INA modelers in their own model. Previously, the ADD team used Adobe Illustrator, PowerPoint, and Visio to create architecture design diagrams, but, as was discussed earlier, they realized the benefit of used the MBSE tool to make one complete model instead of individual diagrams. The figure below (Fig. 6) is an Internal Block Diagram (IBD), currently in production, of a portion of the SCaN Network. Some new methodologies can be seen in this diagram as opposed to diagrams created originally.

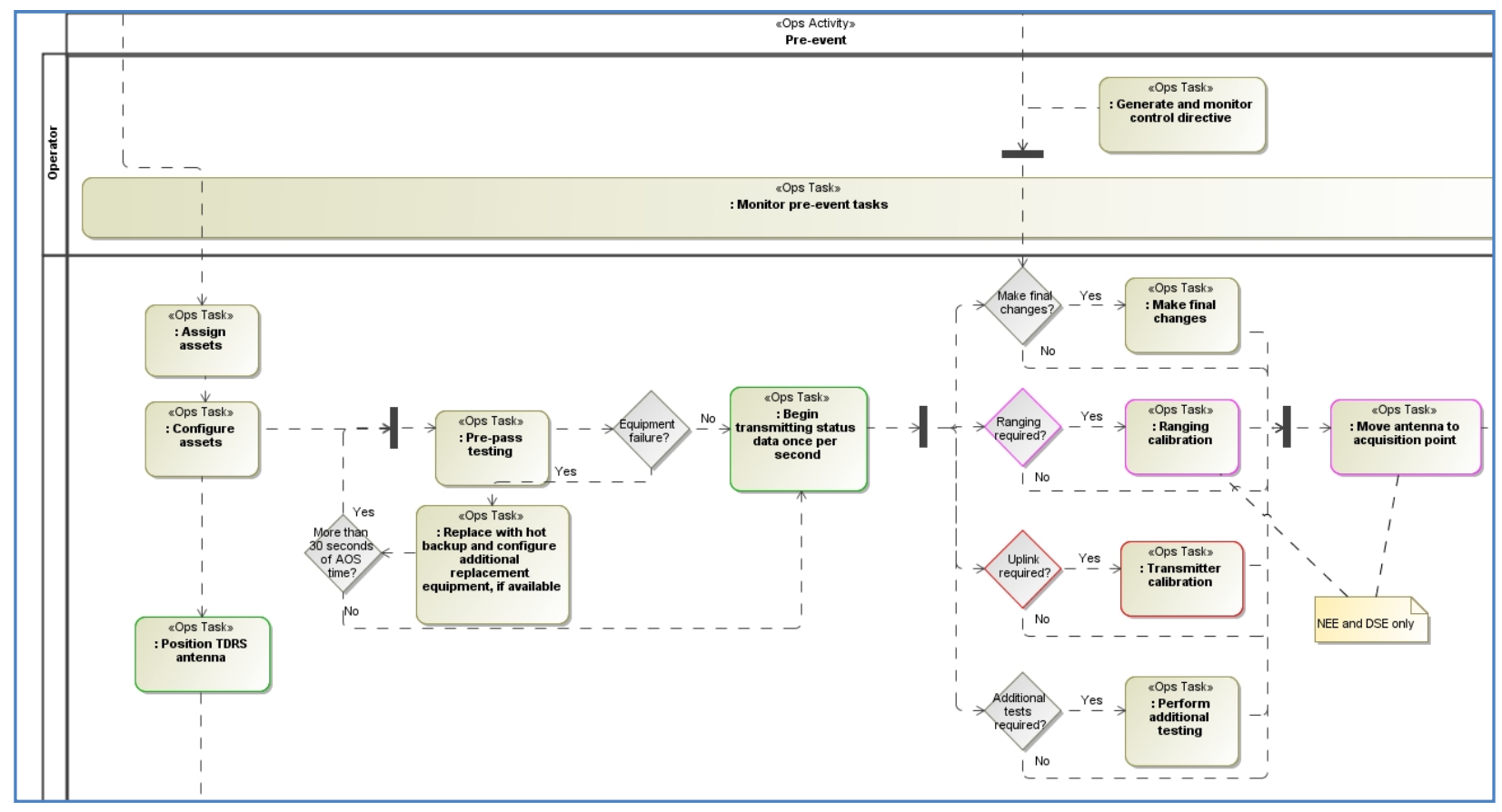

Fig. 5: Network Control Operations Option One OPF

\section{Applying MBSE}

After the early cycles, MBSE usage spread across the SCaN Program. The INA modelers continued to refine modeling standards already in the 


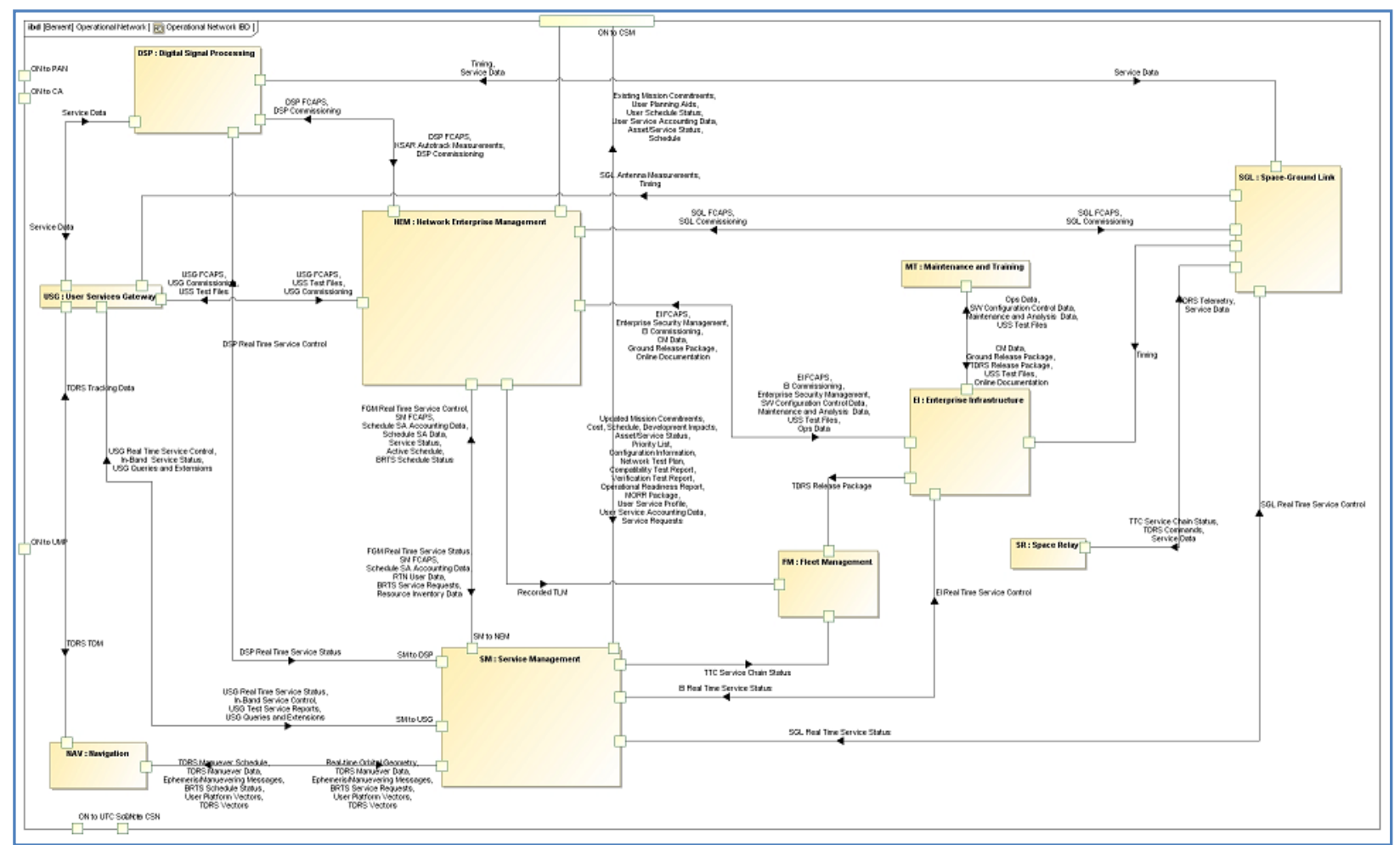

Fig. 6: Operational Network IBD

The Concept of Operations (ConOps) is another SCaN baseline book team that has adopted the MBSE architecture modeling methodologies. Though the ConOps team is in a more infant state when it comes to the complete adoption of MBSE, some of their current work on ConOps scenario definition has been transferred to SysML format. A portion of the Execute Committed Services scenario can be seen in Fig. 7. The full diagram is far too large to fit into this document, but this small section of the diagram shows some of the refined methodology used in current modeling efforts within $\mathrm{SCaN}$.

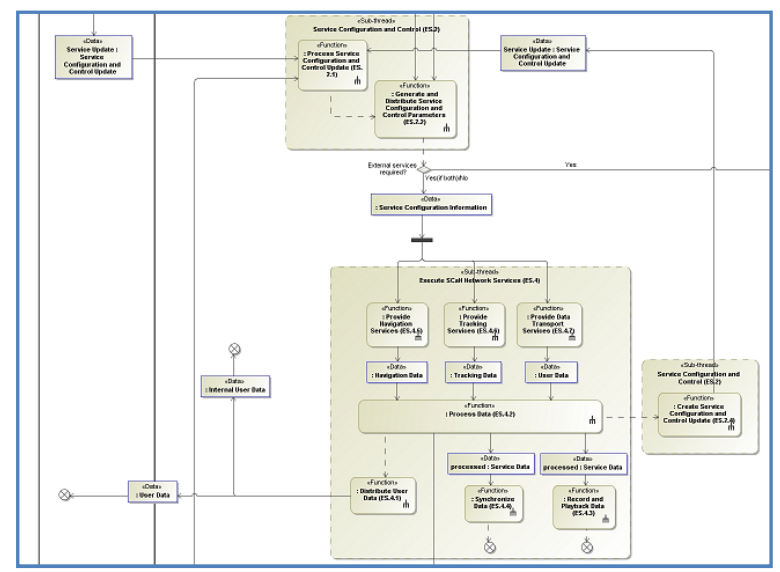

Fig. 7: Execute Committed Services Scenario
In general, the complexity of the models themselves have grown exponentially as the teams have become far more practiced at producing these detailed diagrams using the methodologies started by the original INA modelers. Though the methodologies have been refined and revised over time and the modeling standards within $\mathrm{SCaN}$ teams matured significantly, modeling team members are constantly finding new and better ways to provide information.

IV. The CuRRent Status of the Application OF MBSE

MBSE is now a major part of new activities as they start within SCaN, and many other programs and projects at NASA. Several modelers from every center have become veterans in MBSE methodology. One such project which has started recently is the SCaN Service Portal Project. This project began by inputting Level Two requirements into the MBSE model and then mapping those requirements to functional systems that were designed by the portal team. Several layers of functional and software diagrams were created, along with operational process flows (activity diagrams) for how a user interacts with the portal system. This portal model and the corresponding diagrams formed the basis for development of the portal. The developers began to implement the portal based on the model design and continue to do so. 
The model is used by the Systems Engineering (SE) team for requirements management. Both existing and in-work requirements are stored in the model. These requirements are gathered from the SCaN Network System Requirements Document (SRD) and are mapped to portal functions to ensure that we are not missing any necessary requirements. The mapping is also used to alert the SE team to any possible requirements which may need to be added to the SCaN Network SRD or the SCaN Service Portal requirements. These requirement mapping diagrams and tables have proved invaluable to the SE team and have become the perfect conduit through which the developers and SE can make progress while keeping apprised of each other's status.

MBSE will continue to play a large role in the start of new projects as the SCaN program moves forward with the next integration phase. Some projects will build on the models already started by the INA modelers while some will branch off into separate models. No matter the modeling method is chosen for future projects, the modeling methodologies established in the last few years on the INA trade study will carry on throughout all future modeling tasks.

\section{CONCLUSION}

The INA team working with disparate legacy networks had to understand the complexity of the systems to be modeled before MBSE could be implemented. MBSE allowed the INA team to compare, contrast and analyze multiple complex architecture options. MBSE models have the capability to model infinite levels of software/operational complexity while linking each level to the one above and below. Centralized information (diagrams, definitions) and common terminology made trade study efforts significantly more efficient. MBSE has value when modeling complex systems, the magnitude of that value is being better understood as the implementation process begins.

\section{ACKNOWLEDGEMENT}

The SCaN Program Systems Engineering is supported by numerous team members from the Jet Propulsion Laboratory, Goddard Space Flight Center, Glenn Research Center, and NASA Headquarters. This team would like to give special thanks to the SCaN Integrated Networks Architecture Team, the SCaN Architecture and Requirements Team, and the SCaN Network Subject Matter Experts.

\section{REFERENCES}

[1] "Space Communications and Navigation (SCaN) Network Architecture Definition Document (ADD) Volume 1: Executive Summary", Revision 2, Oct 2011, NASA / $\mathrm{SCaN}$,

https://www.spacecomm.nasa.gov/spacecomm /programs/system_planning/default.cfm

[2] W. Tai, N. Wright, M. Prior, and K. Bhasin, "NASA Integrated Space Communications Network," in Proceedings of SpaceOps Conf., AIAA, Stockholm, Sweden, 2012.

[3] K. Bhasin, B. Golden, J. Roberts, and P. Barnes, "Role of MBSE in NASA's Space Communications Networks," in NASA Project Management (PM) Challenge, Orlando, Florida, 2012.

http://www.nasa.gov/offices/oce/pmchallenge/ library/2012-presentations.html

[4] DoD Architecture Framework Version 2.02, DoD Deputy Chief Information Officer, [online] August 2010, http://dodcio.defense.gov/dodaf20.aspx (Accessed: 15 January 2013)

[5] S. Friedenthal, A. Moore, and R. Steiner, "Practical Guide to SysML", Oxford, Elsevier, 2008. 